EPJ Web of Conferences 113, 04025 (2016)

DOI: $10.1051 /$ epjconf/201611304025

C Owned by the authors, published by EDP Sciences, 2016

\title{
Three-Body Nuclear Systems in Pionless Effective Field Theory
}

\author{
Jared Vanasse $e^{1, a}$ \\ ${ }^{1}$ Department of Physics, Duke University, \\ Durham, NC 27708, USA
}

\begin{abstract}
New perturbative techniques for three-body systems with contact interactions are discussed. Their application to pionless effective field theory $\left(\mathrm{EFT}_{\not t}\right)$ for $n d$ scattering is shown, and their extension to bound states addressed. With the extension to bound states a leading-order $\mathrm{EFT}_{\not k}$ calculation of the triton charge radius and novel treatments of three-body forces are discussed.
\end{abstract}

\section{Introduction}

At low energies $\left(E<m_{\pi}^{2} / M_{N}\right)$ nuclear physics can be described by a theory of short range interactions between nuclei known as pionless effective field theory $\left(\mathrm{EFT}_{\not k}\right)$ (see e.g. [1]). This has been used to great success in the two-body sector, with precision calculations $(<1 \%)$ being performed for $n p \rightarrow d \gamma$ (see e.g. [2]). Progress has also been made in three-body sector with $n d$ scattering but not at the level of precision in the two-body sector (see e.g. [3, 4]). Previously, calculations in the three-body sector of $\mathrm{EFT}_{\not h t}$ have been performed using the partial resummation technique (see e.g. [5]). This technique calculates all diagrams to the order one is working but also includes an infinite subset of higher order diagrams and therefore is not strictly perturbative in the $\mathrm{EFT}_{\not t h}$ power counting. A technique that is strictly perturbative and no more numerically expensive than the partial resummation technique is discussed below and further detail given in Ref. [4]. Its extension to bound state calculations, and the future prospects for precision three-body calculations are also discussed.

\section{Scattering}

The leading-order (LO) $n d$ scattering amplitude in $\mathrm{EFT}_{\not t}$ is given by an infinite sum of diagrams, which can be represented by an integral equation that is solved numerically. The numerical solution of the half off-shell LO $n d$ scattering amplitude is less numerically expensive than solving the full offshell scattering amplitude. Higher order corrections to the $n d$ scattering amplitude in principle require the full off-shell LO $n d$ scattering amplitude. Calculations of higher order corrections using the full off-shell LO scattering amplitude have been performed for cold atoms systems but not for nuclear systems in $\mathrm{EFT}_{\not k}[6]$. To circumvent the need for the full off-shell scattering amplitude the partial resummation technique was created [5]. This technique gives all contributions up to the order one is working but includes an infinite subset of higher order diagrams. Thus this method is not strictly

\footnotetext{
ae-mail: jjv9@phy.duke.edu
} 
perturbative. Also in this method the imaginary part of the quartet (total $\operatorname{spin} S=\frac{3}{2}$ ) $S$-wave phase shift has a negative sign. This is unphysical, and its origin is currently not understood.

A method to calculate higher order corrections strictly perturbatively, that is no more numerically expensive than solving the half off-shell LO scattering amplitude and gives the correct sign for the imaginary part of the quartet $S$-wave phase shift was developed in Ref. [4]. This method was slightly improved upon in Ref. [7], in which the numerical technique for corrections to dibaryon propagators is simplified. In this improved perturbative technique the general $n$th order correction to the scattering amplitude in the quartet channel is given by the integral equation represented in Fig. 1. The final di-

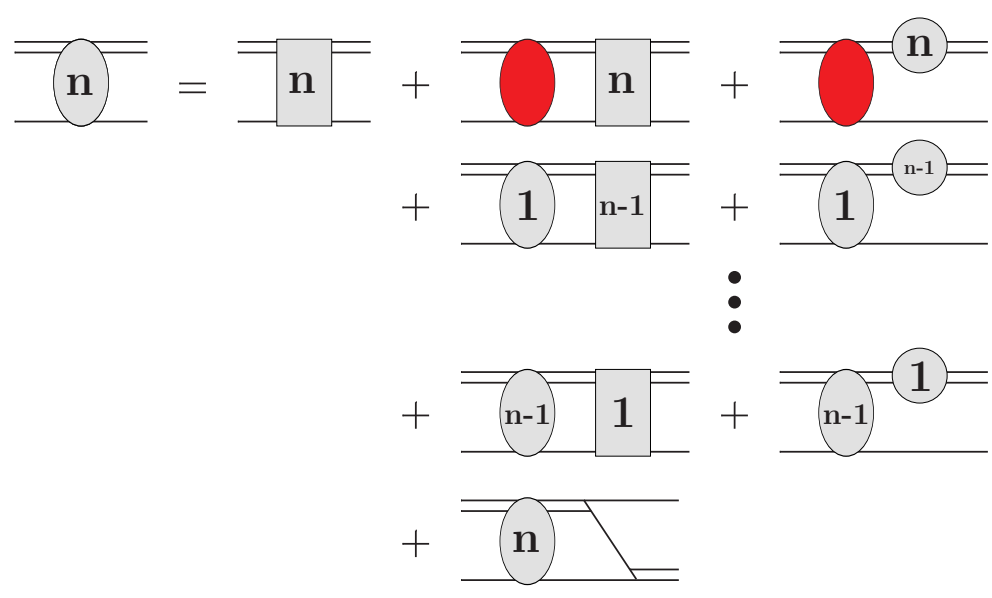

Figure 1. Integral equation for the general $n$th order correction to the quartet channel $n d$ scattering amplitude. The single (double) lines are nucleon (deuteron) propagators, the solid red oval the LO $n d$ scattering amplitude, and the $n$-oval is the $n$th order correction to the $n d$ scattering amplitude. The $n$-circles attached to the deuteron propagators are $n$th order corrections to the deuteron propagator, and the $n$-rectangles are $n$th order "three-body" corrections between neutron and deuteron.

agram, in Fig. 1, is the kernel of the integral equation and is the same kernel for the LO $n d$ scattering amplitude. All preceding diagrams are the inhomogeneous term of the integral equation and depend on lower-order scattering amplitudes. In this technique the kernel of the integral equation is exactly the same at each order, and this simplifies the numerical solution of higher order corrections. Also in this improved technique the diagrams with deuteron corrections become the deuteron wavefunction renormalization corrections in the on-shell limit. Thus in the on-shell limit this improved technique gives the full $n$th order correction including corrections due to the deuteron wavefunction renormalization. Higher order corrections in the doublet channel are given by a similar set of coupled integral equations involving a spin-singlet dibaryon propagator. Additional complications occur in the doublet $S$-wave channel at all orders due to the necessity of three-body forces.

Using these strict perturbative techniques $n d$ scattering has been calculated to next-to-next-toleading-order $\left(\mathrm{N}^{2} \mathrm{LO}\right)$ in $\mathrm{EFT}_{\not h}[4]$. At next-to-leading-order (NLO) and $\mathrm{N}^{2} \mathrm{LO}$ there are effective range corrections to the dibaryon propagators, and at $\mathrm{N}^{2} \mathrm{LO}$ there is a two-body SD-mixing term, which is important since it gives the first non-zero contribution to mixing angles and polarization observables. There is one three-body force at $\mathrm{LO}$ with no derivatives that receives corrections at each order and a new energy dependent three-body force at $\mathrm{N}^{2} \mathrm{LO}$. It was found that at $\mathrm{N}^{2} \mathrm{LO}$ in comparison to potential model calculations that the eigen-phases and mixing angles are mostly reproduced 
well. However, significant differences were found for the $\epsilon_{J}^{\pi}$ mixing angles (mixes different total spin angular momentum but not orbital angular momentum) and the ${ }^{4} P_{J}$ eigen-phase shifts.

\section{Bound States}

The new perturbative method in Ref. [7] can also be applied to three-body bound states, by introducing a triton auxiliary field, $\hat{\psi}$, that interacts with a spin-triplet (spin-singlet) dibaryon field $\hat{t}_{i}\left(\hat{s}_{a}\right)$ and nucleon field $\hat{N}$ via the Lagrangian

$$
\mathcal{L}_{3}=\hat{\psi}^{\dagger}\left(\Omega-h_{2}(\Lambda)\left(i \partial_{0}+\frac{\vec{\nabla}^{2}}{2 M_{N}}+\frac{\gamma_{t}^{2}}{M_{N}}\right)\right) \hat{\psi}+\sum_{n=0}^{2} \omega_{0}^{(n)} \hat{\psi}^{\dagger}\left(\sigma_{i} \hat{N} \hat{t}_{i}-\tau_{a} \hat{N} \hat{s}_{a}\right)+\text { H.c. }
$$

Here, $\gamma_{t}=45.7025 \mathrm{MeV}$, is the deuteron binding momentum. From this formalism the LO triton vertex function [8] can be calculated via an integral equation entirely analogous to the integral equation for $n d$ scattering in the doublet $S$-wave channel with no three-body force. The only difference between these integral equations is the inhomogeneous term. Higher order corrections to the triton vertex function are calculated by integral equations exactly the same as those for perturbative corrections to $n d$ scattering in the doublet $S$-wave channel with no three-body forces, except the $n$th order $n d$ scattering amplitude is replaced by the $n$th order triton vertex function. Using the LO triton vertex function the triton charge radius has been calculated using methods found in Refs. [7, 9] yielding a value of $[1.41 \pm 0.42] \mathrm{fm}[7]$, which is in agreement with the experimental value of [1.755 \pm 0.086$] \mathrm{fm}[10]$. There is also a EFT $\not \not t$ calculation using wavefunction methods that obtains a value of [2.1 \pm 0.6$] \mathrm{fm} \mathrm{[11].}$

This new formalism also offers a novel approach to the treatment of three-body forces. The LO on-shell $n d$ scattering amplitude is given by

$$
T_{\mathrm{LO}}(k)=Z_{\mathrm{LO}} t_{0, N t \rightarrow N t}^{\ell=0}(k, k)+H_{\mathrm{LO}} \frac{1}{1-H_{\mathrm{LO}} \Sigma_{0}(E)} \pi Z_{\mathrm{LO}}\left(G_{0, \psi \rightarrow N t}(E, k)\right)^{2},
$$

where $k$ is the center of mass (c.m.) momentum of the $n d$ system. The function $t_{0, N t \rightarrow N t}^{\ell=0}(k, k)$ is the $n d$ scattering amplitude in the doublet $S$-wave channel with no three-body force, and $G_{0, \psi \rightarrow N t}(E, k)$ is the LO triton vertex function, with $E=\left(\frac{3}{4} k^{2}-\gamma_{t}^{2}\right) / M_{N}$ being the total energy of the system. $Z_{\mathrm{LO}}$ is the $\mathrm{LO}$ deuteron wavefunction renormalization, $H_{\mathrm{LO}}$ the LO three-body force, and $\Sigma_{0}(E)$ a function calculated from integrating over the LO triton vertex function with a LO dibaryon propagator. The novel feature of this expression is that the three-body force is factored out of all numerically determined expressions. Therefore, the three-body force can be fit algebraically using numerically determined quantities yielding

$$
H_{\mathrm{LO}}=\frac{x}{1+x \Sigma_{0}\left(-\gamma_{t}^{2}\right)}
$$

where

$$
x=\frac{-\left(\frac{3 \pi a_{n d}}{M_{N}}+Z_{\mathrm{LO}} t_{0, N t \rightarrow N t}^{\ell=0}(0,0)\right)}{\pi Z_{\mathrm{LO}}\left(G_{0, \psi \rightarrow N t}\left(-\gamma_{t}^{2}, 0\right)\right)^{2}} .
$$

Here the three-body force has been fit to the doublet $S$-wave scattering length, $a_{n d}=.65 \mathrm{fm}$.

\section{Conclusions}

Using these new perturbative methods, $n d$ scattering has been calculated to $\mathrm{N}^{2} \mathrm{LO}$ in $\mathrm{EFT}_{\not t}$ including the SD-mixing term. Comparing with potential model calculations good agreement was found with 
most eigen-phases and mixing angles, with the exception of the $\epsilon_{J}^{\pi}$ mixing angles and ${ }^{4} P_{J}$ eigen-phases. As a result poor agreement was found with low-energy polarization observables in $n d$ scattering. Thus a higher order calculation of $n d$ scattering is necessary to properly address polarization observables. Such a calculation will include shape parameter corrections and two-body $P$-wave contact interactions. Investigating polarization observables using EFT $\not \not t$ is important as there exists the longstanding $A_{y}$ puzzle. This is a discrepancy between theoretical calculations and experimental data for the vector analyzing power, $A_{y}$, which is worse at low energies. However, $\mathrm{EFT}_{\not h t}$ is ideally suited for low energies and offers the prospect of understanding this longstanding puzzle.

Using a triton auxiliary field, a LO EFT $\not \not t$ calculation of the triton charge radius, within the expected LO error, yields agreement with the experimental value. The extension of the perturbative techniques to bound states using the triton auxiliary field gives a straightforward method for calculating higher order corrections to the triton charge radius. This extension also offers a novel numerical treatment and understanding of three-body forces. It allows the LO three-body force to be determined algebraically from numerically determined quantities. Previous methods were more numerically expensive as they were essentially root finding algorithms and required multiple iterations.

Finally, these perturbative techniques work equally well for processes involving external currents and systems with charged particles such as $p d$. Therefore, calculations of $p d$ scattering can be performed, for which there is much more experimental data for polarization observables than $n d$. Investigation of low energy Compton scattering off of the triton and Helium-3 is also possible. For astrophysical applications $p d$ and $n d$ capture at low energies can be considered. Beta decay of the triton can also be addressed and gives a way to fit the $L_{1, A}$ coupling that is an important unknown in $p p$ fusion in $\mathrm{EFT}_{\not k}$ [12]. In short there is a plethora of three-body nuclear physics at low energies that can benefit from these new perturbative techniques and make precision three-body physics in $\mathrm{EFT}_{\not t}$ feasible.

\section{Acknowledgements}

This material is based upon work supported by the U.S. Department of Energy Office of Science, Office of Nuclear Physics, under Award Number DE-FG02-05ER41368. I would also like to thank Roxanne Springer and Thomas Mehen for useful conversations during the course of this work.

\section{References}

[1] S.R. Beane, P.F. Bedaque, W.C. Haxton, D.R. Phillips, M.J. Savage (2000), in At the Frontier of Particle Physics, edited by M. Shiftman, Vol. 1 (World Scientific, River Edge, NJ, 2001).

[2] G. Rupak, Nucl. Phys. A 678, 405 (2000)

[3] H.W. Grießhammer, Nucl. Phys. A 744, 192 (2004)

[4] J. Vanasse, Phys. Rev. C 88, 044001 (2013)

[5] P.F. Bedaque, G. Rupak, H.W. Grießhammer, H.W. Hammer, Nucl. Phys. A 714, 589 (2003)

[6] C. Ji, D.R. Phillips, Few-Body Syst. 54, 2317 (2013)

[7] J. Vanasse, in preparation

[8] R.D. Amado, Phys. Rev. 141, 902 (1966)

[9] P. Hagen, H.W. Hammer, L. Platter, Eur.Phys.J. A49, 118 (2013)

[10] A. Amroun et al., Nucl. Phys. A579, 596 (1994)

[11] L. Platter, H.W. Hammer, Nucl. Phys. A766, 132 (2006)

[12] M. Butler, J.W. Chen, Phys. Lett. B520, 87 (2001) 